\title{
ANSIEDADE E RELIGIOSIDADE DE FAMILIARES DE PACIENTES INTERNADOS EM UNIDADE DE TERAPIA INTENSIVA
}

\author{
Luciana Cardoso Matias \\ Marineia Crosara de Resende \\ (Universidade Federal de Uberlândia - UFU)
}

\begin{abstract}
Resumo
Essa pesquisa, realizada com familiares de pacientes internados numa UTI adulta, teve como objetivos: avaliar o nível de ansiedade apresentado por esses familiares; investigar sua religiosidade; correlacionar religiosidade e ansiedade. Método: Participaram 22 familiares, idade média 44 anos. Responderam em entrevista aos instrumentos: a) Ficha de Informações sócio demográficas; b) Índice Religiosidade DUREL; c) Escala Beck - Ansiedade. Resultados: A partir da análise de dados encontrou-se: ansiedade - nível mínimo; religiosidade -intrínseca (alta), não organizacional e organizacional (no ponto médio). Não houve correlação entre ansiedade e religiosidade. Discussão: É possível inferir que os familiares participantes dessa pesquisa estão ajustados psicologicamente ao evento da internação, utilizando-se de suas crenças pessoais para manterem sua saúde mental, transformando assim a UTI em um local de esperança.
\end{abstract}

Palavras chave: Religiosidade; Ansiedade; UTI Adulto.

\section{Abstract \\ Anxiety and Religiosity in Family Patients Admitted in Intensive Care Units}

This research, carried out with patients' family admitted in adult intensive care unit, aimed to: assess the level of anxiety presented by these relatives; investigate their religiosity; correlate religiosity and anxiety. Participants: 22 family pacients, mean age 44 years old. They answered an interview for the instruments: a) Socio-demographic information; b) DUREL Religiosity Index; c) Beck Scale - Anxiety. Based on the data analysis, it was found: anxiety - minimum level; Religiosity - intrinsic (high), non-organizational and organizational (at the midpoint). There was no correlation between anxiety and religiosity. It is possible to infer that patients' familiars are psychologically adjusted to the hospitalization event. They use personal beliefs to maintain their mental health, transforming the intensive care unit into a place of hope.

Keywords: Religiosity; Anxiety; Intensive Care Unit.

\section{Introdução}

As Unidades de Terapia Intensiva (UTI) são setores hospitalares destinados ao atendimento de pacientes graves ou de risco que dispõe de assistência médica e de enfermagem ininterruptas, com equipamentos próprios, recursos humanos especializados e que tenham acesso a outras tecnologias destinadas ao diagnóstico e a 
terapêutica (Brasil, 1998). Andrade e Resende (2014) pontuam que a hospitalização na UTI acontece, frequentemente, de maneira inesperada, aguda e inadvertida, e que há em torno da UTI representações ameaçadoras que trazem ao paciente e a seus familiares a ideia de gravidade, associada à perda, que pode nem ser real.

No entanto, em sendo uma unidade de pacientes críticos, a UTI produz sentimentos negativos, tanto nos pacientes quanto nos familiares, em razão da sensação de ameaça e risco de morte iminente (Almeida, Aragão, Moura, Lima, Hora, \& Silva, 2009). Frente a essa situação, os familiares podem se sentir desprotegidos, temerosos, confusos e impotentes (Freitas, Kimura, \& Ferreira, 2007).

A família, além de passar pelas alterações causadas pelo processo de adoecer do seu parente e da possibilidade de morte, ainda se defronta com um ambiente até então estranho, com pessoas desconhecidas, onde se executa procedimentos diversificados e, muitas vezes, incompreensíveis, com máquinas assustadoras, que gera expectativas com consequente estado de ansiedade, que pode permanecer até o momento do término da internação (Lunardi Filho, Nunes, Pauletti, \& Lunardi, 2004). A UTI mesmo sendo uma unidade de assistência em saúde dentro de um hospital geral, possui uma arquitetura e um modo de funcionamento próprio (Andrade \& Resende, 2014).

A internação em uma UTI é por si mesma uma condição estressante para o paciente e seus familiares, pois sempre está associada de fato a uma situação de risco (Andrade, \& Resende, 2014) e pode precipitar medo e ansiedade (Lunardi Filho e outros, 2004), decorrentes da preocupação com a morte, das incertezas relacionadas ao prognóstico e ao tratamento, dos conflitos emocionais, da preocupação com a condição financeira, das alterações de papel e da quebra da rotina familiar (Leske, 2002).

Segundo Soares (2007), o cuidado dos familiares é uma das partes mais importantes do cuidado global dos pacientes internados nas UTI. No contexto de um paciente terminal ou no qual as perspectivas de recuperação são muito incertas, esta face do cuidado assume uma importância ainda maior, tendo em vista que o paciente poderá não estar desperto e será preciso manejar e cuidar de seus familiares. Os familiares têm necessidades específicas e apresentam frequências elevadas de estresse, distúrbios do humor e ansiedade durante o acompanhamento da internação na UTI, o que pode persistir após a morte do seu ente querido (Soares, 2007).

A ansiedade é uma reação normal ao estresse e a ameaça ou perigo, que pode ser 
ocasionado pelo medo da morte ou da incapacidade. Ocorre geralmente quando um indivíduo enfrenta uma mudança ou a necessidade de agir de modo diferente do habitual (Almeida e outros, 2009). Para Takei e Schiveletto (2000), a ansiedade constitui um sistema de alerta imediato que previne o organismo contra certos perigos ambientais, às vezes, de forma desproporcional, devendo, nesses casos ser controlada. Para este autor, a ansiedade seria originada do medo e da expectativa quanto ao futuro, podendo ser tão intensa que causaria sintomas inespecíficos, somáticos e distúrbios psíquicos, constituindo-se em motivo de preocupação para os profissionais de saúde.

Os sintomas da ansiedade, durante um longo período de tempo ou acentuados, tornam-se prejudiciais e capazes de causar complicações no funcionamento físico e mental, além de afetar a sociabilidade de um indivíduo (Lunardi filho e outros, 2004). De acordo com Resende, Azevedo, Lourenço, Faria, Alves, Farina, Silva e Oliveira (2011), a ansiedade pode surgir por medo ou insegurança ou como resposta do indivíduo aos eventos externos. Ainda segundo as autoras, pessoas ansiosas apresentam dificuldades em controlar suas emoções e reações, se deixam perturbar facilmente, maximizando efeitos negativos de eventos externos.
Modificações na dinâmica familiar também podem atuar como fator ansiogênico, uma vez que o adoecimento e a consequente internação na UTI podem exigir da família uma reorganização de sua dinâmica, levando a perturbações nas atividades laborais e pessoais de seus membros (Sell, Sell, Nascimento, Padilha, \& Carvalho, 2012). Segundo Lunard Filho e outros, (2004), a ansiedade experimentada pelos familiares pode estabelecer-se como um importante dificultador no contato com a equipe, atrapalhando o relacionamento em decorrência de reações inapropriadas ou fora de contexto.

A ansiedade dos familiares de pacientes internados na UTI pode gerar sentimentos como desconfiança, raiva, insatisfação com o cuidado e inquietude em relação ao tratamento proposto (Leske, 2002), tem como principais fontes, a vulnerabilidade do paciente, possíveis mudanças repentinas do estado geral, falta de informações ou informações excessivas e/ou desencontradas, possíveis trocas de membros da equipe e o acesso restrito a unidade (Lucchesi, Macedo, \& Marco, 2008). Entre as diferentes maneiras com que a família responde a essas vivências estão as estratégias de enfrentamento ativadas na situação de hospitalização em uma UTI, que podem ainda incluir religiosidade (Schleder, Parejo, Puggina, \& 
Silva, 2013). Segundo Souza, Chaves e Silva (2006), a religiosidade pode ser aspecto importante no enfrentamento para quem vivencia uma doença grave de um ente na UTI, uma vez que pode auxiliar na aceitação da dor e do sofrimento ao conferir significados a eles.

Segundo Dalgalarrondo (2008), existe consenso entre os sociólogos, filósofos e psicólogos sociais de que a religiosidade é um importante fator de significação e ordenação da vida, sendo fundamental em momentos de maior impacto na vida das pessoas. A religiosidade e a espiritualidade fazem parte da experiência humana, sendo transversais em qualquer área que envolva a atividade de pessoas, como a arte, a música, a poesia, a guerra, a inspiração, a aspiração, o sacrifício, a moral, a devoção, a contemplação, o conflito, entre outras (Paloutzian, \& Park, 2005).

Oliveira e Junges (2012) relatam que em 1988, a Organização Mundial de Saúde (OMS), incluiu a dimensão espiritual no conceito multidimensional de saúde, remetendo à questões como significado e sentido da vida, e não se limitando a qualquer tipo específico de crença ou prática religiosa. Para a OMS, segundo os autores, a espiritualidade é o conjunto de todas as emoções e convicções de natureza não material, com a suposição de que há mais no viver do que pode ser percebido ou plenamente compreendido.

É importante entender que os termos "espiritualidade" e "religião" estão relacionados, e apesar de, muitas vezes, serem utilizados como sinônimos, apresentam características diferentes. Sommerhalder e Goldstein (2006) relatam que espiritualidade vem do latim, spiritus, que significa "sopro", referindo-se ao sopro da vida e a palavra religião vem do latim, religare, que significa religar, restabelecer a ligação entre Deus e os seres humanos.

A espiritualidade está relacionada com o propósito de vida, com o transcendente (Dalby, 2006), envolve a capacidade de se encantar, de reverenciar e ter gratidão pela vida. É a habilidade de enxergar o sagrado nos fatos comuns, de sentir a intensidade da vida, de ter consciência de uma dimensão sublime, que leva em consideração o próprio ser, os outros, a natureza e a vida (Saad, Masiero, \& Battistella, 2001).

A espiritualidade endereça a uma questão universal relacionada ao significado e ao sentido da vida e à busca humana por valores transcendentais. Também tem sido caracterizada como a capacidade de ter fé, adorar, ver para além das circunstâncias e de transcender o sofrimento (Oliveira \& Junges, 2012). Boff (2006) esclarece que a espiritualidade está 
relacionada com qualidades do espírito humano, tais como, amor e compaixão, paciência e tolerância, capacidade de perdoar, contentamento, noção de responsabilidade, noção de harmonia, que trazem felicidade tanto para a própria pessoa quanto para os outros. Espiritualidade é uma reflexão sobre o significado da vida.

Religiosidade, no entanto, é a expressão ou prática do crente que pode estar relacionada a uma instituição religiosa (Gomes, Farina, \& Dal Foro, 2014). A religião é a crença no direito à salvação que é anunciada pelas tradições da fé, que visam a aceitação de uma realidade metafísica (ideia de paraíso), sendo também associada aos dogmas religiosos, aos rituais e às orações (Boff, 2006).

Tradicionalmente, as religiões buscam responder à questão do significado último da vida. As religiões possuem um código de ética que rege o comportamento e dita os valores morais. Muitas religiões têm como alicerce a crença em um ser supremo ou em um Deus que deve ser adorado, venerado, e as pessoas devem viver de acordo com os seus ensinamentos (Oliveira \& Junges, 2012). Frequentemente religiosidade tem sido associada a um sistema de crença estruturado que remete à questão espiritual.
Para Oliveira, Fitipalddi Neto, Salvi, Camargo, Evangelista, Espinha e Lucchetti (2013), a religiosidade é uma doutrina e um sistema de culto, compartilhados por um grupo de pessoas, com características comportamentais, sociais, doutrinárias e com valores específicos. A religião também pode favorecer uma forma de expressar a espiritualidade, porque possui sistemas organizados, como rituais e cerimônias, que, junto com a inserção na comunidade religiosa, proporcionam o sentimento de pertencimento, podendo servir de base para o significado da existência. Algumas crenças religiosas também podem despertar sentimentos negativos, como medo, culpa e angústia, especialmente aquelas doutrinas que pregam o ser supremo como punitivo, vingativo, que vigia todos os comportamentos. (Sommerhalder \& Goldstein, 2006).

Mesmo distintas espiritualidade e religião podem se relacionar e conviver, mas sem que uma dependa necessariamente da outra (Boff, 2006). A manifestação da espiritualidade e o envolvimento em religiões organizadas têm sido relacionados ao aumento do senso de propósito de vida, a maior resiliência e resistência ao estresse relativo às doenças (Lawler \& Younger, 2002 citado por Panzini \& Bandeira, 2007). $\mathrm{O}$ uso de crenças e comportamentos religiosos para o enfrentamento de 
situações negativas, conhecido como coping religioso (Panzini \& Bandeira, 2007; Freitas, et al., 2007) pode estar presente nos familiares no momento da hospitalização em uma UTI, como um recurso auxiliar para o enfrentamento do estresse e manutenção da resiliência (Schleder e outros, 2013) e o controle da ansiedade. Segundo Bousso, Poles, Serafim e Miranda (2011), a religiosidade pode ser ativada pela família na sustentação do equilíbrio emocional e no controle da ansiedade, pois, embora não resolva a situação instantaneamente, permite a família renovar suas energias, identificar recursos para lidar com incertezas e encontrar respostas para

\section{Método}

\section{Participantes}

Participaram da pesquisa, 22 familiares em $1^{\circ}$ ou $2^{\circ}$ grau de pacientes internados numa UTI Adulto, com idade entre 22 e 59 anos (média 44; DP = 12,92), sendo a maioria do sexo masculino $(54,5 \%)$, casado (50,0\%), filho(a) do familiar (50,0\%), católico $(50,0 \%)$. As características demográficas podem ser vistas na Tabela 1.

Tabela 1 - Distribuição de frequência das características sociodemográficas dos participantes $(n=22)$. questionamentos relacionados ao adoecimento e até mesmo a morte.

A partir do exposto realizou-se a presente investigação junto a familiares de pacientes internados numa UTI Adulto de um hospital da rede pública de saúde com os objetivos de:

$\checkmark$ Avaliar o nível de ansiedade
apresentado por familiares de
pacientes internados em UTI;
$\checkmark$ Investigar a presença da
religiosidade em familiares de
pacientes internados em UTI;
$\checkmark$ Correlacionar religiosidade e
ansiedade em familiares de
pacientes internados em UTI.

\begin{tabular}{|c|c|c|}
\hline Variável & $\begin{array}{l}\text { Porcentag } \\
\text { em }\end{array}$ & $\begin{array}{l}\text { Frequên } \\
\text { cia }\end{array}$ \\
\hline \multicolumn{3}{|l|}{ Gênero } \\
\hline Masculino & 54,55 & 12 \\
\hline Feminino & 45,45 & 10 \\
\hline Total & 100,0 & 22 \\
\hline \multicolumn{3}{|l|}{ Idade } \\
\hline$<30$ & 18,18 & 4 \\
\hline $30-39$ & 18,18 & 4 \\
\hline $40-49$ & 18,18 & 4 \\
\hline $50-59$ & 45,45 & 10 \\
\hline Total & 100,0 & 22 \\
\hline \multicolumn{3}{|l|}{ Estado Civil } \\
\hline Solteiro & 36,36 & 8 \\
\hline Casado & 50,00 & 11 \\
\hline Divorciado & 13,64 & 3 \\
\hline Total & 100,0 & 22 \\
\hline $\begin{array}{l}\text { Parentesco com } \\
\text { paciente }\end{array}$ & $\mathbf{o}$ & \\
\hline Pai/Mãe & 27,27 & 6 \\
\hline Filho(a) & 50,00 & 11 \\
\hline Irmão(ã) & 22,73 & 5 \\
\hline
\end{tabular}




\begin{tabular}{lll}
$\begin{array}{l}\text { Total } \\
\text { Orientação } \\
\text { religiosa/espiritual }\end{array}$ & 100,0 & 22 \\
Católica & 50,0 & 11 \\
Espírita & 18,18 & 4 \\
Evangélica & 18,18 & 4 \\
Outra (Cristão) & 4,55 & 1 \\
Não possui & 9,09 & 2 \\
Total & 100,0 & 22 \\
\hline
\end{tabular}

A maioria dos familiares pesquisados são parentes em $1^{\circ}$ grau, contabilizando $77,27 \%$ do total, sendo que destes a maioria $36,36 \%$ são filhos de pacientes internados na UTI Adulto e $18,18 \%$ são mães dos pacientes.

Constatou-se que $72,7 \%$ dos familiares exercem suas funções profissionais no setor terciário da economia, $9,1 \%$ são do setor primário, outros $9,1 \%$ são estudantes, $9,1 \%$ são do lar e 4,55\% aposentado.

Em relação à religião, verificou-se que $50 \%$ dos familiares entrevistados se dizem católicos, 18,18\% se dizem Espíritas, 18,18\% Evangélicos, 9,9\% disseram não pertencer a nenhuma denominação religiosa e $4,55 \%$ se autodenominaram cristãos.

\section{Instrumentos}

a) Ficha de Informações sócio demográficas - Questionário para coleta de dados sobre idade, gênero, renda mensal, profissão, ocupação, estado civil, parentesco com o paciente e tipo de arranjo domiciliar.

b) Índice de Religiosidade DUREL (Moreira-Almeida, Peres, Aloe, Lotufo Neto, \& Koenig, 2008; Taunay, Gondim, Macêdo, Moreira-Almeida, Gurgel, Andrade, \& Carvalho, 2012): é uma escala que compreende três das dimensões de religiosidade que mais se relacionam com saúde: organizacional (RO), nãoorganizacional (RNO) e religiosidade intrínseca (RI). É composta por cinco itens e na análise dos resultados, as pontuações nas três dimensões (RO, RNO e RI) são analisadas isoladamente. A primeira questão abrange a RO, questionando a frequência de idas a igrejas, templos ou outros encontros religiosos, com opções de resposta numeradas de 1 ("Mais do que uma vez por semana") a 6 ("Nunca"). O segundo item trata da RNO (frequência de atividades religiosas individuais, como preces e leitura da Bíblia), também com opções de resposta numeradas de 1 ("Mais do que uma vez ao dia") a 6 ("Raramente ou nunca"). As três últimas questões abordam a RI, em que o entrevistando deve responder entre alternativas de 1 ("Totalmente verdade para mim") a 5 ("Não é verdade") o quanto cada frase a respeito de crenças ou experiências religiosas se aplica em sua vida.

c) Escala de Ansiedade de Beck - BAI (Cunha, 2001), teste psicológico que visa 


\section{LUCIANA CARDOSO MATIAS, MARINEIA CROSARA DE RESENDE}

medir a intensidade de sintomas de ansiedade. O inventário é constituído de 21 itens, que são afirmações descritivas de sintomas de ansiedade, e que devem ser avaliados pelo participante com referência a si mesmo e levando em consideração a última semana numa escala de quatro pontos (de "absolutamente não" a "gravemente: dificilmente pude suportar").

\section{Procedimentos para coleta de dados}

Após aprovação do projeto de pesquisa pelo Comitê de Ética em Pesquisa foi feito contato com o familiar do paciente internado na UTI - Adulto do HCUFU no horário da visita, solicitando a participação na pesquisa. Foram explicados os objetivos da pesquisa, o caráter voluntário da participação, a garantia de sigilo dos dados coletados. Para a aplicação foram convidados a participar os 22 primeiros familiares que comparecerem na UTI Adulto no horário da visita e que aceitarem participar da pesquisa; individualmente em entrevista e antes cada participante foi convidado a ler e a assinar o Termo de Consentimento Livre e Esclarecido. A aplicação dos instrumentos aconteceu após o horário de visita e do boletim médico em uma sala reservada dentro da própria unidade, que teve duração máxima de 30 minutos para cada familiar.

\section{Procedimentos para análise de dados}

Para descrever o perfil da amostra segundo as variáveis em estudo, foram feitas tabelas de frequência das variáveis categóricas, com valores de frequência absoluta (n) e percentual (\%) e estatísticas descritivas das variáveis numéricas, com valores de média, desvio padrão, valores mínimos e máximos, mediana e quartis. Para comparação das variáveis numéricas entre os grupos de níveis de estresse foram utilizados os testes de Mann-Whitney (para duas categorias) e de Kruskal-Wallis (para três ou mais categorias), devido ao tamanho reduzido da amostra e à ausência de distribuição normal dos escores. Para analisar a relação entre as variáveis numéricas foi utilizado o coeficiente de correlação de Spearman. O nível de significância adotado para os testes estatísticos foi de $5 \%$, ou seja, $\mathrm{p}<0,05$.

\section{Procedimentos éticos}

O projeto foi aprovado pelo Comitê de Ética da Universidade Federal de Uberlândia: Parecer $n^{\circ} 1.776 .683 / 2016$. Os procedimentos do presente estudo seguiram as normas regulamentadoras de pesquisa com seres humanos do Conselho Nacional de Saúde (Resolução 466/2012). Todos os 
participantes receberam informações acerca dos objetivos da pesquisa, das atividades a serem desenvolvidas e de seus direitos, antes de assinarem o Termo de

\section{Resultados e Discussão}

Com relação ao nível de ansiedade, os familiares de pacientes internados na UTI adulta apresentam os seguintes resultados: 50,0\% nível mínimo, 22,73\% nível leve, 18,18\% nível moderado e 9,09\% nível grave.

A ansiedade, de acordo com Batista e Oliveira (2005), é um termo que se refere a uma relação de impotência, conflito existente entre a pessoa e o ambiente ameaçador, e os processos neurofisiológicos decorrentes dessa relação. Para estes autores, a ansiedade constitui a experiência subjetiva do organismo numa condição trágica, que surge na medida em que o indivíduo, frente a uma situação, não pode enfrentar às exigências de seu meio e, sente uma ameaça à sua existência ou aos valores que considera essenciais. A ansiedade pode ser situacional (quando está associada a determinados acontecimentos), como por exemplo, a internação de um familiar numa UTI ou ser constante na vida do indivíduo.

Ter um ente querido em situação de necessidade de cuidados em uma UTI
Consentimento Livre e Esclarecido. A identidade de todos os participantes foi mantida em sigilo.

sempre gera algum tipo de preocupação, assim, é necessário considerar o contexto pelo qual a emoção ocorre, além das suas características individuais para determinar se as manifestações clínicas são desproporcionais à intensidade e a duração dos fatores desencadeantes (Castro, Quarantini, Batista-Neves, Kraychete, Daltro, \& Miranda-Scippa, 2006). E, apesar da ansiedade ser um sentimento vago e desagradável de medo, apreensão, caracterizado por tensão ou desconforto derivado de antecipação de perigo, de algo desconhecido ou estranho, sob o qual não se tem controle (Castillo, Recondo, Asbahr, \& Manfro, 2000), neste estudo, a maioria dos familiares dos pacientes internados na UTI Adulto apresentaram nível mínimo ou leve de ansiedade.

Portanto, os baixos níveis de ansiedade apresentados pelos participantes dessa pesquisa podem indicar que os familiares estão vivenciando sua manifestação de dor, impotência e separação de seus entes queridos dentro dos parâmetros de ansiedade normal. Para Kaplan, Sadock e Sadock (2007), se pode falar de ansiedade normal quando esta é 


\section{LUCIANA CARDOSO MATIAS, MARINEIA CROSARA DE RESENDE}

considerada como um sinal de alerta; e como advertência de uma ameaça externa ou interna é considerada adaptativa. Ainda segundo os autores, na ansiedade normal há reação proporcional à ameaça objetiva, não envolvendo repressão ou outros mecanismos de conflito intrapsíquico, nem a ativação de mecanismos de defesa neurótica, e pode ser enfrentada construtivamente pela percepção consciente ou pode ser aliviada se a situação objetiva for alterada.

Constatou-se, nessa pesquisa, que em relação à religiosidade, os familiares de pacientes internados em UTI apresentam alta Religiosidade intrínseca (média 1,52; $\mathrm{DP}=0,74)$, apresentam Religiosidade não organizacional (média 2,27; DP $=0,88$ ) e Religiosidade organizacional no ponto médio (média 3,27; DP = 1,32).

A prevalência da religiosidade intrínseca significa que vivem a religião como uma crença que interiorizaram e praticam na sua totalidade de maneira interna, privada. Pessoas com motivação religiosa intrínseca colocam suas crenças religiosas em primeiro lugar, vivendo de acordo com seus preceitos, numa atitude de comprometimento e busca de sentido da vida (Duarte \& Wanderley, 2011).

De acordo com Dias (2011), os valores intrínsecos têm sido correlacionados com altos níveis de saúde mental, conduzindo frequentemente à transcendência, em que o indivíduo se considera participante de um plano divino e experiencia gratidão ao contemplar o universo, encontra-se mais fortemente correlacionada com o sentido na vida. Uma religião internalizada, intrinsecamente motivada, e construída sob a crença de que existe um maior significado na vida, uma relação segura com Deus e um sentido de ligação espiritual têm implicações positivas no bem-estar. (Dias, 2011).

Com a prevalência da Religiosidade intrínseca, dimensão subjetiva, do quanto e como o indivíduo percebe a importância da religião em sua vida (Koenig \& Büssing, 2010), seguida por Religiosidade NãoOrganizacional, os participantes relatam realizar atividades religiosas individuais, que podem ser realizadas a partir da privacidade do lar como orações, leituras e programas de $\mathrm{TV}$ e/ou rádio e que independem da interação com outras pessoas (Koenig \& Büssing, 2010), sendo $68,2 \%$ diariamente, $9,1 \%$ mais que uma vez ao dia, $13,7 \%$ duas ou mais vezes por semana, $4,5 \%$ uma vez por semana e $4,5 \%$ poucas vezes por mês.

\section{Com relação a Religiosidade} Organizacional, que ficou no ponto médio, a participação e frequência em igrejas, templos ou encontros religiosos, aquela relacionada a um componente social 
(Koenig \& Büssing, 2010), apenas 36,4\% dos familiares frequentam uma ou mais vezes por semana, $18,2 \%$ duas ou três vezes ao mês, $27,3 \%$ algumas vezes ao ano. Enquanto 13,6\% referiu frequentar tais encontros uma vez por ano ou menos e $4,5 \%$ dos familiares nunca frequentam. Estes dados sugerem que a importância que a religião ocupa na vida destas pessoas precisa ser mensurado pelo significado atribuído as práticas religiosas individuais.

Pelos resultados, verificou-se que não houve correlação entre os escores de espiritualidade/religiosidade e os níveis de ansiedade (Ver Tabela 2 e 3).

Tabela 2. Correlações entre os escores das escalas religiosidade e ansiedade

\begin{tabular}{lllll}
\hline & RO & RNO & RI \\
\hline Ansiedade & $\mathrm{r}=$ & 0.26272 & 0.12817 & 0.07787 \\
\hline & $\mathrm{p}=$ & 0.2375 & 0.5697 & 0.7305 \\
\hline
\end{tabular}

* $\mathrm{r}=$ coeficiente de correlação de Spearman; $\mathrm{P}=$ Valor-P; $\mathrm{n}=$ número de sujeitos $(\mathrm{n}=22)$.

Tabela 3. Comparação dos escores de religiosidade entre os níveis de ansiedade.

\begin{tabular}{|c|c|c|c|c|c|}
\hline $\begin{array}{l}\text { Ansiedad } \\
\text { e }\end{array}$ & $\begin{array}{l}\text { Variáve } \\
\text { l }\end{array}$ & $\mathbf{N}$ & $\begin{array}{l}\text { Médi } \\
\text { a }\end{array}$ & DP & Valor-p* \\
\hline \multirow{9}{*}{$0-10$} & & 1 & & 1.3 & \\
\hline & RO & 1 & 2.91 & 0 & $\mathrm{p}=0.210$ \\
\hline & & 1 & & 0.7 & \\
\hline & RNO & 1 & 2.18 & 5 & $\mathrm{p}=0.691$ \\
\hline & & 1 & & 0.5 & \\
\hline & RI & 1 & 1.45 & 4 & $\mathrm{p}=0.807$ \\
\hline & & 1 & & 1.2 & \\
\hline & RO & 1 & 3.64 & 9 & \\
\hline & RNO & $\begin{array}{l}1 \\
1\end{array}$ & 2.36 & $\begin{array}{l}1.0 \\
3\end{array}$ & \\
\hline
\end{tabular}

\begin{tabular}{llll} 
& 1 & & 1.0 \\
$\mathrm{RI}$ & 1 & 1.58 & 7 \\
\hline
\end{tabular}

\begin{tabular}{|c|c|c|c|c|c|}
\hline Ansiedad & Variáve & $\mathbf{N}$ & Médi & DP & Valor- \\
\hline \multirow{6}{*}{$0-10$} & & 1 & & 1.3 & \\
\hline & RO & 1 & 2.91 & 0 & 0.551 \\
\hline & & 1 & & 0.7 & \\
\hline & RNO & 1 & 2.18 & 5 & 0.659 \\
\hline & & 1 & & 0.5 & \\
\hline & RI & 1 & 1,45 & 4 & 0.932 \\
\hline \multirow{6}{*}{$11-19$} & & & & 1.4 & \\
\hline & RO & 5 & 3.80 & 8 & \\
\hline & & & & 1.5 & \\
\hline & RNO & 5 & 2.40 & 2 & \\
\hline & & & & 1.6 & \\
\hline & RI & 5 & 1.80 & 1 & \\
\hline \multirow{6}{*}{$20-30$} & & & & 1.2 & \\
\hline & RO & 4 & 3.75 & 6 & \\
\hline & & & & 0.5 & \\
\hline & RNO & 4 & 2.50 & 8 & \\
\hline & & & & 0.3 & \\
\hline & RI & 4 & 1.42 & 2 & \\
\hline
\end{tabular}

\begin{tabular}{lllll}
\hline & & & 1.4 \\
$31-63$ & RO & 2 & 3.00 & 1 \\
\cline { 2 - 5 } & RNO & 2 & 2.00 & 0.0 \\
& RI & 2 & 1.33 & 7
\end{tabular}

* Valor-P referente ao teste de Mann-Whitney para comparação das variáveis entre 2 grupos.

** Valor-P referente ao teste de Kruskal-Wallis para comparação das variáveis entre 3 ou mais grupos.

Diferente dos dados encontrados nessa pesquisa, Melo, Sampaio, Souza e Pinto (2015) relatam que diferentes estudiosos defendem que o envolvimento religioso reduz a ansiedade ao oferecer respostas que permitem uma organização de conflitos emocionais, estabelecem um sistema de orientação moral e ética, além de desestimular práticas consideradas destrutivas para a saúde de uma forma geral. 


\section{LUCIANA CARDOSO MATIAS, MARINEIA CROSARA DE RESENDE}

Alguns autores associam a religiosidade à autoestima, desejo de viver e diminuição da ansiedade, do estresse e da depressão (Tusca-Molina, Herrera, Sosa, Martinez, Arjona, Cueto, \& Manrique, 2003). As práticas religiosas, que podem ajudar a manter a sanidade mental e prevenir doenças mentais, porque elas influenciam psicodinamicamente, auxiliando o indivíduo a manejar a ansiedade, medos, frustrações, raiva, sentimentos de inferioridade, desânimo e isolamento (Murakamil \& Campos, 2012).

Vários estudos já evidenciaram a religiosidade como estratégia de enfrentamento em situações estressoras, diminuindo os níveis de ansiedade. Apesar de não ter sido encontrado nesse estudo, relação entre religiosidade e ansiedade, acredita-se ser de suma importância a continuidade de pesquisas que investiguem essas variáveis, correlacionando-as com outras variáveis psicológicas, como traços de personalidade, valores humanos, resiliência, bem-estar subjetivo, ajustamento psicológico, suporte social entre tantas outras.

Ao que os resultados indicam, é possível inferir que os familiares participantes dessa pesquisa estão ajustados psicologicamente ao evento da internação à UTI, apresentando nível mínimo de ansiedade e não tendo influência de sua religiosidade. E mesmo que não tenha sido encontrada correlações entre as variáveis, os familiares se utilizam de suas crenças pessoais (religiosidade intrínseca) para manterem sua saúde mental.

A UTI sempre foi considerada um local angustiante, potencialmente gerador de ansiedade nos familiares e nos pacientes internados, em função de viverem momentos de tensão ao se separarem de seus entes queridos e sempre enfrentarem a possibilidade de morte. No entanto, o baixo nível de ansiedade apresentado pelos familiares de pacientes internados na UTI Adulto aqui pesquisado, indica que parece estar havendo uma mudança na percepção que se tem desse local.

$\mathrm{Na}$ atualidade a UTI tem se apresentado como um espaço de recuperação da saúde e da vida. A presença de uma equipe multiprofissional, com acolhimento inclusive para os familiares, principalmente por profissionais e residentes da psicologia, tem contribuído para a substituição de medos e de incertezas por esperança de recuperação e retorno à vida cotidiana. 


\section{Referências}

Almeida, A. S., Aragão, N. R. O., Moura, E., Lima, G. C, Hora, E. C., \& Silva, L. A. S. M. (2009). Sentimentos dos familiares em relação ao paciente internado na unidade de terapia intensiva. Revista Brasileira de Enfermagem, 62(6), 844-849. DOI 10.1590/S003471672009000600007.

Andrade, R. Z., \& Resende, M. C. (2014). Avaliação dos agentes estressores e da resiliência em pacientes internados na Unidade de Terapia Intensiva. Perspectivas em Psicologia, 10, 194-213.

Batista, M. A., \& Oliveira, S. M. S. V. (2005). Sintomas de ansiedade mais comuns em adolescentes. Psic (on-line), 6(2), 43-50.

Boff, L. (2006). Um caminho de transformação. Rio de Janeiro: Sextante.

Bousso, R. S, Poles, K, Serafim, T. S., \& Miranda, M.G. (2011). Crenças religiosas, doença e morte: perspectiva da família na experiência de doença. Revista da Escola de Enfermagem da USP, 45(2), 397-403. DOI 10.1590/S0080-62342011000200014.

Brasil. (1998). Ministério da Saúde, Lei 3.432, de 12 de agosto de 1998. Dispõe sobre critérios de classificação para as unidades de tratamento intensivo. Brasília: Ministério da Saúde; 1998.

Castillo, A. R. G. L., Recondo, R., Asbahr, F. R., \& Manfro, G. G. (2000). Transtornos de ansiedade. Revista Brasileira Psiquiatria, 22(2), 20-23. DOI 10.1590/S151644462000000600006.

Castro, M. M. C., Quarantini, L., Batista-Neves, S., Kraychete, D. C., Daltro, C., MirandaScippa, A. (2006). Validade da Escala Hospitalar de Ansiedade e Depressão em Pacientes com Dor Crônica. Revista Brasileira de Anestesiologia, 56(5), 470-477. DOI 10.1590/S0034-70942006000500005.

Cunha, J. A. (2001). Manual de versão em português das escalas Beck. São Paulo: Casa do Psicólogo.

Dalby, P. (2006). Is there a process of spiritual change or development with ageing? A critical review of research. Aging \& Mental Health,10(1), 4-12. DOI 10.1080/13607860500307969.

Dalgalarrondo, P. (2008). Religião, Psicopatologia e saúde mental. Porto Alegre: Artes Médicas.

Dias, C. M. B. L. C. (2011). Religiosidade intrínseca e extrínseca: implicações no bem-estar subjectivo de adultos de meia idade. Dissertação de Mestrado. Universidade de Lisboa: Faculdade de Psicologia. Portugal. 
Duarte, F. M., \& Wanderley, K. S. (2011). Religião e espiritualidade de idosos internados em uma enfermaria geriátrica. Psicologia: Teoria e Pesquisa,27(1), 49-53. DOI 10.1590/S0102-37722011000100007.

Freitas, K.S., Kimura, M., \& Ferreira, K.A.S.L. (2007). Necessidades de familiares de pacientes em unidades de terapia intensiva: análise comparativa entre hospital público e privado. Rev Latino-am Enfermagem, 15(1), 84-92. DOI 10.1590/S0104-11692007000100013.

Gomes, N. S., Farina, M., \& Dal Foro, C. (2014). Espiritualidade, Religiosidade e Religião: reflexões de conceitos em artigos psicológicos. Revista de Psicologia da IMED, 6(2), $107-$ 112. DOI 10.18256/2175-5027/psico-imed.v6n2p107-112.

Kaplan, H. I., Sadock, B. J., \& Sadock, V. A. (2007). Compêndio de Psiquiatria: Ciência do Comportamento e Psiquiatria Clínica. 9 ${ }^{\mathrm{a}}$ ed. Porto Alegre: Artmed.

Koenig, H. G., Büssing, A. (2010). The Duke University Religion Index (DUREL): a five-item measure for use in epidemiological studies. Religions, 1(1), 78-85. DOI 10.3390/rel1010078.

Lawler, K. A., Younger, J. W. (2002). Theobiology: an analysis of spirituality, cardiovascular responses, stress, mood, and physical health. Jounal Relig Health 41(4), 347-362. DOI 10.1023/A:1021126510680.

Leske, J. S. (2002). Interventions to decrease family anxiety. Crit Care Nurse, 22(6), 61-65.

Lunardi Filho, D., Nunes, A. C., Pauletti, G, \& Lunardi, V. L. (2004). As manifestações de ansiedade em familiares de pacientes internados em Unidades de Terapia Intensivas gerais. Família. Saúde e Desenvolvimento, 6(2), p.100-109.

Lucchesi, F., Macedo, P. C. M., \& Marco, M. A. (2008). Saúde mental na unidade de terapia intensiva. Revista da SBPH, 11(1), 19-30.

Melo, C. F. Sampaio, I. S., Souza, D. L. A., \& Pinto, N. S. (2015). Correlação entre religiosidade, espiritualidade e qualidade de vida: uma revisão de literatura. Estudos $e$ Pesquisas em Psicologia, 15(2), 447-464. DOI 10.12957/epp.2015.17650.

Moreira-Almeida, A., Peres, M. F., Aloe, F., Lotufo Neto, F., \& Koenig, H. G. (2008). Versão em português da Escala de Religiosidade da Duke: DUREL. Archives of Clinical Psychiatry (São Paulo), 35(1), 31-32. DOI 10.1590/S0101-60832008000100006.

Murakamil, R., \& Campos, C. J. G. (2012). Religião e saúde mental: desafio de integrar a religiosidade ao cuidado com o paciente. Revista Brasileira de Enfermagem, 65(2), 361367. DOI 10.1590/S0034-71672012000200024.

Oliveira, M. R., \& Junges, J. R. (2012). Saúde mental e espiritualidade/religiosidade: a visão de psicólogos. Estudos de Psicologia, 17(3), 469-476. DOI 10.1590/S1413294X2012000300016.

Oliveira, G. R., Fitipalddi Neto, J. F., Salvi, M. C., Camargo, S. M., Evangelista, J. L., Espinha, D. C. M. E. \& Lucchetti, G. (2013). Saúde, espiritualidade e ética: A percepção dos 
pacientes e a integralidade do cuidado. Revista da Sociedade Brasileira de Clínica Médica, $11(2), 140-144$.

Paloutzian, R. F., \& Park, C. L. (2005). Handbook of the psychology of religion and spirituality. New York: The Guilford Press.

Panzini, R. G., \& Bandeira, D. R. (2007). Coping (enfrentamento) religioso/espiritual. Archives of Clinical Psychiatry (São Paulo), 34 (Suppl. 1), 126-135. DOI 10.1590/S010160832007000700016.

Resende, M. C., Azevedo, E. G. S., Lourenço, L. R., Faria, L. S., Alves, N. F., Farina, N. P., Silva, N. C., \& Oliveira, S. L. (2011). Saúde mental e ansiedade em agentes comunitários que atuam em saúde da família em Uberlândia (MG, Brasil). Ciência \& Saúde Coletiva, 16(4), 2115-2122. DOI 10.1590/S1413-81232011000400011.

Saad, M., Masiero, D., \& Battistella, L. Z. (2001). Espiritualidade baseada em evidências. Acta Fisiátrica, 8(3), 107-112. DOI 10.5935/0104-7795.20010003.

Schleder, L. P., Parejo, L. S., Puggina, A. C., \& Silva, M. J. P. (2013). Espiritualidade dos familiares de pacientes internados em unidade de terapia intensiva. Acta Paulista de Enfermagem, 26(1), 71-78. DOI 10.1590/S0103-21002013000100012.

Sell, C. T., Sell, B. T., Nascimento, E. R. P., Padilha, M. I., Carvalho, J. B. (2012). Alterações na dinâmica familiar com a hospitalização em unidade de terapia intensiva. Rev Enferm UERJ, 20(4), 488-492.

Soares, M. (2007). Cuidando da Família de Pacientes em Situação de Terminalidade Internados na Unidade de Terapia Intensiva. RBTI, 19(4), 481-484. DOI 10.1590/S0103$507 X 2007000400013$.

Sommerhalder, C., \& Goldstein, L. L. (2006). O papel da espiritualidade e da religiosidade na vida adulta e na velhice. Em E. V. Freitas, L. Py, F. A. X. Cançado \& M. L. Gorzoni (Eds.). Tratado de Geriatria e Gerontologia (2a ed), (pp.1307-1315). Rio de Janeiro: Guanabara Koogan.

Schleder, L. P., Parejo, L. S., Puggina, A. C., \& Silva, M. J. P. (2013). Espiritualidade dos familiares de pacientes internados em unidade de terapia intensiva. Acta Paulista de Enfermagem, 26(1), 71-78. DOI 10.1590/S0103-21002013000100012.

Souza, S. R. O. S., Chaves, S. R. F., \& Silva, C. A. (2006). Visita na UTI: um encontro entre desconhecidos. Revista Brasileira de Enfermagem, 59(5), 609-613. DOI 10.1590/S003471672006000500003.

Takei, E. H., \& Schiveletto, S. (2000). Como diagnosticar e tratar ansiedade. Revista Brasileira de Medicina, 57(7), 665-666.

Taunay, T. C. D'E., Gondim, F. A. A., Macêdo, D. S., Moreira-Almeida, A., Gurgel, L. A., Andrade, L. M. S., \& Carvalho, A. F. (2012). Validação da versão brasileira da escala de religiosidade de Duke (DUREL). Archives of Clinical Psychiatry (São Paulo), 39(4), 130135. DOI 10.1590/S0101-60832012000400003. 
Tuesca-Molina, R., Herrera, N. F., Sosa, A. M., Martinez, F. O., Arjona, Y. P., Cueto, J. P., \& Manrique, I. S. (2003). Los grupos de socializacion como factor protector contra la depresion en personas ancianas. Baranquilla, Colombia. Revista Espanhola de Salud Pública, 77, 595-604. DOI 10.1590/S1135-57272003000500008.

\section{As autoras:}

Luciana Cardoso Matias possui graduação em Psicologia pela Faculdade Pitágoras de Uberlândia (2014) e graduação em Ciências Biológicas pela Universidade Federal de Uberlândia (2003).

Marineia Crosara de Resende é Psicóloga, Professora do Instituto de Psicologia, Tutora/orientadora da Psicologia no Programa de Residência Multiprofissional em Saúde pela Universidade Federal de Uberlândia. Universidade Federal de Uberlândia, Instituto de Psicologia. Av. Pará, 1720, Campus Umuarama, CEP 38405382 - Uberlândia, MG - Brasil, Telefone: (34) 3218-2185

\section{Endereço para correspondência:}

Profa. Dra. Marineia Crosara de Resende

Instituto de Psicologia da Universidade Federal de Uberlândia

Av. Pará, 1720, bloco 2C, Campus Umuarama

CEP: 38405-382 - Uberlândia - MG.

E-mail: marineia@ipsi.ufu.br

Recebido em: 20/04/2018.

Aprovado em: 10/06/2018. 